\title{
Quasi-elastic and inelastic inclusive electron scattering from an oxygen jet target
}

\author{
M. Anghinolfi, M. Ripani, M. Battaglieri, R. Cenni, P. Corvisiero, \\ A. Longhi, V.I. Mokeev, G. Ricco, M. Taiuti, A. Teglia, A. Zucchiatti \\ Physics Department, University of Genova and Istituto Nazionale di Fisica Nucleare - \\ Sezione di Genova Via Dodecaneso 33, I-16146 Genova (Italy)
}

\author{
N. Bianchi, A. Fantoni, P. Levi Sandri, V. Lucherini, V. Muccifora, \\ E. Polli, A. Reolon, P. Rossi \\ Istituto Nazionale di Fisica Nucleare - Laboratori Nazionali di Frascati \\ P. O. Box 13, I-00044 Frascati (Italy)
}

\author{
S. Simula \\ Istituto Nazionale di Fisica Nucleare - Sezione Sanità, \\ Viale Regina Elena 299, I-00161 Rome (Italy)
}

\begin{abstract}
The results of an experiment on inclusive electron scattering from an oxygen jet target, performed in a wide range of energy and momentum transfer covering both quasi-elastic and $\Delta(1232)$ resonance regions, are reported. In the former region the theoretical predictions, obtained including effects of nucleon-nucleon correlations in both initial and final states, give a good description of the experimental data. In the inelastic region a broadening as well as a damping of the resonant part of the cross section with respect to the free nucleon case is observed. The need of more detailed calculations including nuclear structure effects on the electroproduction cross section of nucleon resonances is highlighted.
\end{abstract}

PACS 25.30.F — Keywords: inclusive electron scattering, nuclear medium effects.

\footnotetext{
${ }^{a}$ To appear in Nucl. Phys. A.
} 


\section{Introduction}

Investigation of inclusive electron scattering processes $A\left(e, e^{\prime}\right) X$ off nuclei at high momentum transfer can provide relevant information on the nuclear wave function and, at values of energy transfer above pion production threshold, on excitation, propagation and decay of nucleon resonances in nuclear medium. To this purpose several experiments have been performed [1-4] showing that, at values of squared four-momentum transfer $Q^{2} \sim 0.1-1$ $(\mathrm{GeV} / \mathrm{c})^{2}$, the inclusive cross section, as a function of the energy transfer $\omega$, is characterized by two broad and prominent peaks which are clearly related to the processes of quasi-elastic (QE) scattering and $\Delta(1232)$ resonance electroproduction. As a matter of fact, the centroids of the two peaks are approximately located at $W \cong M=938 \mathrm{MeV}$ and $W \cong M_{\Delta}=1232$ $\mathrm{MeV}$ respectively [5], where $W \equiv \sqrt{M^{2}+Q^{2}(1 / x-1)}$ is the invariant mass produced on a free nucleon at rest and $x=Q^{2} / 2 M \omega$ is the Bjorken scaling variable. Thus, the general features of the inclusive cross section for the $A\left(e, e^{\prime}\right) X$ reaction are expected to be dominated by the virtual photon absorption on a quasi-free nucleon. However, such a simple picture holds only for kinematical conditions close to the centre of the QE peak (i.e., at $x \sim 1$ ), where the overall behaviour of the total cross section can be accounted for by calculations based on the plane wave Impulse Approximation (IA) using a mean-field description of the nuclear structure [6]. It should be pointed out that reaction mechanisms different from the quasi-free one can contribute to the total cross section at kinematics corresponding to both sides of the QE peak. In the low energy side $(x>1)$ the inclusive cross section is sensitive both to nuclear binding effects (i.e., to high momentum and high removal energy components generated in the nuclear wave function by nucleon-nucleon (NN) short-range and tensor correlations) and to Final State Interaction (FSI) effects between the knocked-out nucleon and the residual nuclear system (see for example ref. [7]). In the high energy side of QE peak $(x<1)$ contributions to the total cross section arising from non-nucleonic degrees of freedom and inelastic nucleonic channels become, in addition, relevant. In kinematical regions corresponding to the $\Delta(1232)$ resonance excitation, the picture that emerges from existing experimental data [1-4] can be summarized as follows: i) both width and height as well as location of the $\Delta(1232)$ peak are modified by medium effects but, at the same time, the total cross section per nucleon scales with the mass number $A$; ii) the inclusive cross sections measured in the dip region at low $Q^{2}\left(Q^{2} \leq 0.2(\mathrm{GeV} / \mathrm{c})^{2}\right)$ between the $\mathrm{QE}$ and $\Delta(1232)$ peaks, are higher than the theoretical prediction, even when the effects resulting from pion production and the corrections due to Meson Exchange Currents (MEC) are included $[8,9]$. Medium effects, such as Fermi motion, nuclear binding, Pauli blocking and pion reabsorption, are thought to be responsible of the modifications of the width of $\Delta(1232)$ resonance and of its location in energy. It should also be pointed out that in the case of real photons the excitation of nucleon resonances with masses above the $\Delta(1232)$ seems to be sizably suppressed in nuclei, leading to a damping of such resonances from the total nuclear photoabsorption cross section in this region [10]. Thus, the use of virtual photons to investigate the excitation of nucleon resonances in nuclei could be of great relevance, providing information on how baryon structure is affected by the presence of other nucleons. In brief, measurement of inclusive cross section for $A\left(e, e^{\prime}\right) X$ processes at intermediate values of $Q^{2}\left(\sim 0.1-1(\mathrm{GeV} / \mathrm{c})^{2}\right)$ still represents a powerful tool to investigate both the nuclear 
structure and medium-dependent modifications of electroexcitation of the most prominent nucleon resonances.

The aim of this paper is to report on an inclusive electron scattering experiment performed at ADONE storage ring at Frascati using an oxygen jet target and a shower calorimeter. The apparatus allowed the simultaneous measurement of inclusive cross section in a wide range of values of energy transfer, ranging from quasi-elastic peak to kinematical regions beyond the $\Delta(1232)$ resonance, at values of three-momentum transfer $q \equiv|\mathbf{q}|$ up to $\sim 800 \mathrm{MeV} / \mathrm{c}$. This paper is organized as follows. The experimental apparatus is described in section 2. The data analysis, pair production subtraction and radiative corrections are discussed in detail in section 3. A comparison of the experimental data with theoretical predictions both in $\mathrm{QE}$ and in $\Delta(1232)$ resonance regions is presented in section 4 . The main conclusions are summarized in section 5 .

\section{Experimental apparatus}

The experiment was performed at ADONE, the Frascati storage ring, using 0.5 through 1.5 $\mathrm{GeV}$ electrons scattered from a clustered jet target [11] placed on a straight section of the ring. At the interaction point the jet was a $6 \mathrm{~mm}$ spot whereas the electron beam had a dimension of $3 \mathrm{~mm}$ FWHM. At each run the stored current was initially $50 \mathrm{~mA}$, the beam lifetime was 40 minutes for the typical $\sim 1 \mathrm{ng} / \mathrm{cm}^{2}$ target density and the luminosity varied from $10^{-31} \mathrm{~cm}^{-2} \mathrm{~s}^{-1}$ down to a factor 5 lower at the end of each measurement. Due to this exponential decrease an on-line monitor of the luminosity was necessary and a detection of Møller electrons was used to this purpose, as described later on. The scattered electrons were measured at $32^{0}, 37.1^{0}, 83^{0}$ at different energies from 0.5 to $1.5 \mathrm{GeV}$ with a scintillation detector composed by a front part which allowed mass separation and angular definition and by a rear part consisting of BGO crystals to measure the energy of the scattered electrons and to improve their separation from proton and pion background [12]. Despite the common use of magnetic spectrometers in electron scattering experiments, our calorimeter provided a valid alternative for this apparatus since an energy resolution of few percent was required, still sufficient to separate the broad structures of QE and $\Delta(1232)$ peaks. Different components were present in this detector:

1. a telescopic system of small plastic scintillators to discriminate against neutral particles and to define the solid angle, which could be varied from 4 to $30 \mathrm{msr}$ depending on the position of the detector (forward or backward angles respectively);

2. an aerogel Cherenkov detector with a refraction index $n=1.045$ to separate electrons from pions with momentum lower than $0.5 \mathrm{GeV} / \mathrm{c}$ and protons. The counter efficiency was measured [13] using electron pairs produced by the Frascati tagged photon beam incident on a radiator and it turned out to be $97 \%$;

3. a BGO pre-shower of $2.5 \mathrm{~cm}$ thickness to improve the separation of electrons from charged heavier particles: as a matter of fact, the energy deposited in this device is markedly different for the radiating electron with respect to an ionizing heavier particle; 
4. the shower calorimeter consisting of 20 BGO crystals of 24 radiation lengths thickness contained in a carbon fiber housing which is part of a $4 \pi$ electromagnetic calorimeter [14]. Besides giving the sum of the energies deposited in each crystal, the granularity of this detector was used as a further test to distinguish electrons from the other particles.

The detector temperature was monitored by several thermocouples and kept constant by a temperature control system, whereas LEDs were used to monitor possible PMT gain variations. Proton events as measured in the pre-shower $(d E / d x)$ and in the calorimeter $(E)$ produced a narrow line [15], providing a precise complementary method to monitor such fluctuations, always limited within $3 \%$ and corrected in the off-line analysis.

Due to this multiparametric information a rejection better than $99 \%$ of hadrons against electrons was obtained, the electron total identification efficiency being $95 \%$ in all our energy range. This experimental equipment was also designed to achieve a complete electromagnetic shower absorption in the calorimeter and therefore no response function unfolding procedure had to be applied to the collected data. The energy of scattered electrons was determined as the sum of the energies released in the calorimeter and in the preshower, the energy loss in the thin plastics being negligible. The final energy resolution was moderate $(\sim 2.5 \%$ FWHM for $1.5 \mathrm{GeV}$ electrons) but definitely sufficient to separate QE and $\Delta(1232)$ peaks.

A $(d E / d x-E)$ plastic telescope was used to detect the monokinetic Møller electrons scattered at $30^{\circ}$ with respect to the beam. This simple device provided the luminosity monitor: its response was independent of $a$ ) luminosity fluctuations, $b$ ) energy of the electron beam and c) low energy electromagnetic background close to the beam line which was measured to be less than $1 \%$ of Møller events.

\section{Data analysis and radiative corrections}

For each run the electron yield was obtained as a function of the kinetic energy ranging from detection threshold $(\sim 100 \mathrm{MeV})$ up to the tail of QE peak. In order to obtain good statistics, the spectra corresponding to different runs at the same kinematics were summed. In this analysis the following procedure was adopted:

1. the reproducibility of energy calibration of each spectrum was checked within $1 \%$ by determining the centroid of the proton line in the plot of energies released in the preshower and in the calorimeter [15];

2. all the events above threshold giving a non-zero signal in the Cherenkov counter were then normalized to the luminosity of each run dividing it by the number of detected Møller electrons. The plot of fig. 1 shows the result of this procedure for the spectra collected at $880 \mathrm{MeV}$ beam energy and $32^{0}$ scattering angle: the fluctuations are purely statistical and there is no evidence of a dependence on the injected current and the jet density fluctuations;

3. data relative to each partial run were summed; then, counts were binned in $25 \mathrm{MeV}$ energy intervals which correspond to the absolute FWHM energy resolution for 1.5 
$\mathrm{GeV}$ electrons. The absolute value of the cross section was finally obtained using the efficiency and solid angle of the Møller detector as well as the Møller cross section.

Radiative corrections were calculated with a computer code [16] and subtracted from the spectra. In our case $t^{2}$ effects, $t$ being the target thickness, were completely negligible and corrections were therefore applied to account for elastic radiation tail, multiple soft photon emission and continuum. The radiation tail for the elastic peak was subtracted avoiding the peaking approximation and using the exact formula given in ref. [17] and the measured elastic form factors for ${ }^{12} \mathrm{C}$ and ${ }^{16} \mathrm{O}$ [18]. The calculation of the elastic tail was checked by a direct comparison to already published results [19]. Contribution of this effect, as can be seen in fig. 2, is present in the high energy transfer region only; in the same figure the other corrections discussed later on are also reported. Following ref. [17] multiple soft photon emission was accounted for, whereas for the continuum contribution two different approaches were applied for the hard photon emission before or after the inelastic nuclear scattering. In the first case radiatively corrected data at lower incident energies as well as lower momentum transfer were needed. For this purpose we used an interpolation of our data when available, or the result of a phenomenological model [20] which describes the nuclear response function with reasonable accuracy in the region of interest. The radiation of hard photons after the inelastic nuclear scattering needed, on the other hand, an ad hoc evaluation due to the presence of a non magnetic apparatus [16]. Since the angle between final electron and radiated hard photon was well inside the angular acceptance, the total energy released in the detector was still the same as if the scattered electron had not radiated. Therefore, this correction was applied at the actual energy transfer and produced a smooth few percent reduction of the cross section. In fig. 2 the spectrum of $880 \mathrm{MeV}$ electrons scattered from ${ }^{16} \mathrm{O}$ at $32^{0}$ is reported at different steps of the analysis: the small difference between raw and radiatively corrected data in the QE peak is due to a partial compensation between multiple soft photon radiation process and the term describing the hard photon emission.

As far as pair production contamination is concerned, in the present measurement no experimental subtraction of this contribution was possible because of the absence of a magnetic field. Terms depending on $t^{2}$ were, however, completely negligible, whereas the contribution linearly depending on $t$ was calculated following a well established procedure [21] which indicate no presence of such a background in the QE region as shown in fig. 2. At higher energy transfer the pair production contamination becomes sizable and it was found to be in agreement with previously measured pair production spectra [1, 22]. This background was therefore subtracted from the data up to transferred energies where the sum of both radiative and pair production corrections was below $40 \%$ of the measured cross section. At very low energy transfer, contributions to the cross section from elastic scattering off nucleus or inelastic transitions to bound or quasi-bound excited states are possible; however, in our three-momentum transfer range such contributions were completely negligible [23].

Even though, due to the low luminosity, both electronic dead time and pulses pile-up were negligible, some efficiency loss on the four-fold coincidence of the electron detector could not be excluded. Moreover, our target was not able to produce a hydrogen jet of reasonable density; therefore a comparison with the parametrization of all the previous $\mathrm{H}\left(e, e^{\prime}\right) X$ measurement in order to check absolute normalization was impossible. Therefore, to check our apparatus performances and data analysis we carried out some of our measurements in 
the same kinematical conditions of published data [19] on ${ }^{16} \mathrm{O}$, namely 540 and $730 \mathrm{MeV}$ beam energy at $37.1^{0}$ scattering angle. In both cases we found that a factor 1.19 had to be applied to our data in order to reproduce the spectra of ref. [19]: in fig. 3 the comparison of our measurement and those of ref. [19] performed at $540 \mathrm{MeV}$ and $37.1^{0}$ shows good agreement between the two data sets; we found an analogous agreement at $730 \mathrm{MeV}$ beam energy. The good quality of data of ref. [19] and the complete overlap of their and our cross sections give us confidence both in our data analysis and in the normalization procedure. The stability of the apparatus was checked performing at the beginning and at the end of each run (typically 7 days) the above mentioned normalization measurements: no variations exceeding statistical uncertainties were found.

The total systematic error of $4.5 \%$ in the QE peak was obtained from the quadratic sum of the accuracy of radiative corrections (3\%), calibration procedure as deduced from ref. [19] (3\%), electron beam energy (1\%) and uncertainty on the solid angle of the detector placed at different angles (1\%). A value lower than $6 \%$ was instead evaluated in the $\mathrm{D}_{13}(1520)$ resonance region were both pair production subtraction and radiative corrections become sizable.

\section{Results and comparison with theoretical predictions}

\subsection{Quasi-elastic peak}

In this section the experimental results obtained for the radiatively corrected cross sections will be shown and compared with the theoretical predictions for the QE region described in ref. [24]. In order to clarify the role played by FSI, the results corresponding to the IA and those including an estimation of FSI will be considered. In this approach the cross section for inclusive process $A\left(e, e^{\prime}\right) X$ is written in the following form:

$$
\frac{d^{2} \sigma}{d E_{e^{\prime}} d \Omega_{e^{\prime}}}=\sigma_{0}+\sigma_{1}
$$

where the contributions from different final nuclear states have been explicitly separated out, namely $\sigma_{0}$ describes the transition to ground and one-hole states of the $(A-1)$-nucleon system and $\sigma_{1}$ thetransition to more complex highly excited configurations. As it is known, within the IA, the evaluation of the inclusive cross section requires the knowledge of the nucleon spectral function $P(k, E)$, which represents the joint probability to find in a nucleus a nucleon with momentum $k \equiv|\mathbf{k}|$ and removal energy $E$. In presence of ground state NN correlations $P(k, E)$ can be written as $P(k, E)=P_{0}(k, E)+P_{1}(k, E)$, where the indexes 0 and 1 have the same meaning as in eq. (1), i.e. $P_{0}$ includes ground and one-hole states of the $(A-1)$-nucleon system and $P_{1}$ more complex configurations (mainly $1 \mathrm{p}-2 \mathrm{~h}$ states) which arise from $2 \mathrm{p}-2 \mathrm{~h}$ excitations generated in the target ground state by NN correlations. Whereas $P_{0}$ depends on the nucleon momentum distribution of the single particle states, for the correlated part $P_{1}$ we will make use of the model developed in refs. [25, 26], which involves the basic two-nucleon configurations generating the high momentum and high removal energy behaviour of the spectral function. Finally, besides the two spectral functions $P_{0}$ and $P_{1}$, the cross section of eq. (1) depends on $\sigma_{e N}$, describing the scattering of the electron by an off-shell nucleon as computed in [27]. 
Comparison of our results in the QE region with the described IA approach is reported in fig. 4 by the dashed line. The experimental data of ${ }^{16} \mathrm{O}$ were collected at $32^{0}$ scattering angle and different beam energies. The inclusion of transitions to highly excited configurations accounted for by $P_{1}$ is not sufficient to explain the experimental cross section. At the QE peak the calculations largely overestimate the measured strength especially for the data at $700 \mathrm{MeV}$ beam energy, whereas an opposite effect is observed in all the collected spectra in the low energy transfer region corresponding to $x \geq 1.5$.

In order to account for such differences, both single and two nucleon rescattering have been included, following the approach of ref. [24]. This approach treats consistently the effects of NN correlations in both initial and final nuclear states: in particular, a locally correlated NN pair with its centre of mass apart from the spectator $(A-2)$ nucleus is considered $[25,26]$ and the two emitted nucleons are allowed to rescatter elastically in the final states. It should be pointed out that the approach of ref. [24] has been positively checked against SLAC data [28] both for light and complex nuclei at $x>1$ and high momentum transfer $\left(Q^{2}>1(\mathrm{GeV} / \mathrm{c})^{2}\right)$. In the present paper the same approach is applied to the calculation for lower values of $Q^{2}\left(0.1<Q^{2}<0.6(\mathrm{GeV} / \mathrm{c})^{2}\right)$ and the result, together with our experimental data, is represented in fig. 4. The inclusion of such FSI is sizable: from the QE peak the strength is correctly moved to both low and high energy transfer regions greatly improving the agreement with the experimental data and extending the $Q^{2}$ interval where this theoretical approach can describe the data.

\subsection{The resonance region}

At energy transfer higher than the QE peak, the inclusive cross section is dominated by the pion-nucleon resonance, the $\Delta(1232)$ and, for our highest beam energy, by the less prominent $\mathrm{N}(1520)$. This is clearly evident from fig. 5, where some of our inclusive spectra of ${ }^{16} \mathrm{O}$ are reported up to $W \approx 1500 \mathrm{MeV}$. In the following analysis the inclusive cross sections on ${ }^{12} \mathrm{C}$ from ref. [4] were also used. The data have been divided by the nuclear mass $A$ but the slightly different kinematics (scattering angle and $Q^{2}$ ) do not allow a direct comparison of the measured strength for the two different nuclei. However, when the normalization to the virtual photon flux was also performed, a response well within the total uncertainty was obtained as shown in fig. 6 for two measurements with similar $Q^{2}$ in the $\Delta(1232)$ peak.

Different approaches have been attempted in order to describe the excitation mechanism in this region. An evaluation of the pion electroproduction cross section on single nucleon taking into account resonant, non-resonant (Born) terms and final state interactions [29] was extended to finite nuclei [30] including the two-body $\gamma \mathrm{NN} \rightarrow \mathrm{NN}$ mechanism which is assumed to be dominated by the coupling to the $\pi \mathrm{NN}$ intermediate state. In order to reproduce the experimental data, the medium effects on the propagation of $\Delta(1232)$ were included by changing the $\Delta(1232)$ self-energy from its value in the free space. The $\Delta$-hole approach [31] carefully describes the resonant part of the reaction mechanism: this model, successfully applied to the description of pion- and photon-induced nuclear reactions in the resonance region, includes dynamicaleffects like Pauli blocking and pion multiple scattering. These calculations have been compared to the experimental data on light nuclei at a relatively low momentum $\left(Q^{2} \leq 0.15(\mathrm{GeV} / \mathrm{c})^{2}\right)$ in the $\Delta(1232)$ peak: in both cases the predicted cross section turns out to be lower than the data from the dip to the $\Delta(1232)$ peak by $15 \%$. 
Our data were, instead, collected in a higher momentum transfer region $\left(0.1 \leq Q^{2} \leq 0.5\right.$ $\left.(\mathrm{GeV} / \mathrm{c})^{2}\right)$ where the impulse approximation is expected to dominate: in order to find possible medium modifications to the single nucleon strength, we compared the experimental data to a simple calculation where only Fermi motion effects were taken into consideration. The main steps of our analysis are here summarized.

Starting from the well known Brasse parametrization of experimental electron scattering cross section on free proton [32], we fitted more recent $\mathrm{H}\left(e, e^{\prime}\right)$ data [4], measured in kinematical conditions very similar to ours, by a small tuning of the original parameters of the fit. Then, following the prescription of ref. [33], the result of our parametrization was folded on the nucleon momentum distribution given in ref. [25] to obtain the inelastic structure functions in the nucleus. The same distribution was assumed both for our data in ${ }^{16} \mathrm{O}$ and for ${ }^{12} \mathrm{C}$ data of ref. [4] and no difference in the virtual photon absorption on proton $\sigma_{p}$ and neutron $\sigma_{n}$ was taken into account. The result of this calculation is reported in fig. 5 together with both our measurements in ${ }^{16} \mathrm{O}$ and ref. [4] data in ${ }^{12} \mathrm{C}$ for different beam energies and scattering angles. The QE peak contribution, evaluated as described in the previous section, was directly summed to the inelastic part and the result compared to the data: this comparison immediately shows that the resonance structure has a broader shape than the calculation, whereas a damping of the inelastic strength with respect to the single nucleon seems to be required at the higher $Q^{2}$ values. This is even more evident for ${ }^{12} \mathrm{C}$ data (fig. $5 c$ and $d$ ) measured with less statistical uncertainty. It is important to note that these data have been taken in exactly the same kinematical conditions of the $\mathrm{H}\left(e, e^{\prime}\right)$ reaction we used to evaluate the single particle inelastic strength.

This result is consistent with the known fact that the $\Delta(1232)$ resonance in nuclei is broadened by an additional width beyond the natural decay width and Fermi motion [2]: to single out this nuclear effect on the resonance cross section it was therefore necessary to develop first a suitable model for $\Delta(1232)$ excitation on free nucleon. We assumed a relativistic approach where the correct treatment of the kinematics ensures that the $\gamma N \Delta$ transition form factor is evaluated at the proper $Q^{2}$. Thus we used the $\Delta$ propagator of the Rarita-Schwinger theory, whereas the Peccei Lagrangian $[8,34,35]$ provided the $\gamma N \Delta$ vertex. The $\gamma N \Delta$ coupling constant is usually chosen to reproduce the resonant channel $\mathrm{M}^{+}$of $\gamma \mathrm{N} \rightarrow \pi N$ reaction. Since, however, the Peccei Lagrangian provides an abnormally high contribution for the Coulomb multipole of about $15 \%$, we simply rescaled the peak of the same amount to account for $\mathrm{M}^{+}$transitions only. Moreover, $\Delta(1232)$ cannot be considered as stable. We thus added in the denominator of the propagator its width in the vacuum (dependent on energy and momentum to account, for instance, for threshold effects $[36])$. Its strong coupling constant and form factor were finally fixed by the elastic $\pi \mathrm{N}$ cross section.

As far as higher energies are concerned, at $W \approx 1500 \mathrm{MeV}$ two resonances are observed in the nucleon: $\mathrm{N}(1520)$ and $\mathrm{N}(1535)$ with a full width similar to $\Delta(1232)$. Since this 15 $\mathrm{MeV}$ mass difference is far below the energy resolution of our apparatus, we considered only the $\mathrm{N}(1520)$ resonance which was assumed to have the same dependence of the cross section on the energy transfer as the $\Delta(1232)$. The strength was determined from $\mathrm{H}\left(e, e^{\prime}\right)$ data as reported, in one example, in fig. 7 ; the continuous curve is the result of the modified Brasse fit we used to determine the inelastic part whereas the dashed lines represent the separated contributions to the total strength: our calculated $\Delta(1232)$ excitation curve, the $\mathrm{N}(1520)$ 
peak - whose amplitude was kept as a free parameter - and a phenomenological non-resonant background. For the latter the expression

$$
B(W)=\alpha\left(W-W_{0}\right)^{\beta}
$$

was assumed where $W_{0}$ is the pion electroproduction threshold and $\alpha, \beta$ are free parameters. In all the spectra we have assumed the parameter $\beta$ to be linearly dependent on the four momentum value at the pion electroproduction threshold $Q_{t h}^{2}$ :

$$
\beta=0.70 Q_{t h}^{2}-0.10
$$

whereas the values of $\alpha$ were sensitive to the specific kinematical conditions.

In order to extend this analysis to the nucleus, the two resonances and the continuous background were separately folded with the momentum distribution and shifted by the average nucleon binding energy. In view to highlight possible medium modification to the quasi-free picture, the model amplitude and width have been therefore adjusted to fit the observed data in the high energy transfer region both for ${ }^{16} \mathrm{O}$ and ${ }^{12} \mathrm{C}$. As a first step we broadened both resonances by an additional width (in quadrature) up to a maximum value corresponding to twice the experimental value reported in ref. [2]. Result of this procedure is reported for our and ref. [4] data in fig.8. Here the two broadened resonances and the continuous background are separately plotted, while the total sum including QE peak calculation is represented by the continuous curve: whereas for the low beam energy data this widening accounts for the observed strength, at higher energies (higher $Q^{2}$ ) an excess of strength which can not be reduced, at least for ${ }^{12} \mathrm{C}$ data, by a further spreading out of resonances is nevertheless present. This could be an indication that, accordingly to the real photon case [37], a proper suppression factor must be simultaneously applied to original resonance amplitude. In fig. 9 the thin curve corresponds to the result of this procedure: it is now evident that a proper suppression factor to the resonant strength can account for the experimental data.

However, the result of this analysis is not unambiguous, especially in N(1520) region where the data are limited to $W \leq 1500 \mathrm{MeV}$. In the same figure we have, in fact, also reported the computed strength when both the broadened resonances and the remaining inelastic part are damped by the same factor, obtaining a very similar result. The limited discrepancy observed in the high energy transfer side of the QE peak might be ascribed to the folding with momentum distribution instead of spectral function in the inelastic part calculation.

The results of the analysis are plotted in fig. 10 and summarized in table I: here $\sigma$ represents the extra width applied to the resonances, $a$ and $b$ are the cross section suppression factors for the $\Delta(1232)$ and $\mathrm{N}(1520)$ resonances respectively, $c$, instead, represents the overall suppression factor when both the resonant and the non-resonant part are simultaneously changed. This analysis shows that the extra width $\sigma$ necessary to reproduce the shape of $\Delta(1232)$ peak is quite independent from the kinematics, whereas the suppression factors seem to be stronger as $Q^{2}$ increases. If, for the $\Delta(1232)$ resonance, this factor approaches to unity at low $Q^{2}$, for the $\mathrm{N}(1520)$ resonance a damping is always observed giving some sort of continuity with respect to the real photon case. The consistency of the extra width and suppression factor for ${ }^{12} \mathrm{C}$ and ${ }^{16} \mathrm{O}$ data suggests a similar behaviour of the response function 
of the two nuclei in the analyzed momentum range. Even if the quoted errors are statistical only, corresponding to a unit change on $\chi^{2}$, the inclusion of the systematic error $(6 \%$ for our data and 3\% for the ref. [4] ones) does not seem to significantly change the results of this analysis.

A major source of indetermination could, instead, be introduced by a possible difference between proton $\sigma_{p}$ and neutron $\sigma_{n}$ absorption strengths. A parametrization which takes into account this difference [38] gives, in fact, $\sigma_{n} / \sigma_{p} \cong 0.76$ in the kinematical range of our and ref. [4] data. Using this parametrization, the suppression factors found in the previous analysis are reduced. This fit, however, is the result of the extrapolation of proton and deuteron data measured at higher $Q^{2}\left(Q^{2}>1(\mathrm{GeV} / \mathrm{c})^{2}\right)$ and its accuracy in our kinematical range might be questionable. In order to give a more quantitative evaluation of the protonneutron difference, the following considerations can be done. Due to the isospin structure of the interaction, the proton and neutron helicity amplitudes relative to the $\Delta(1232)$ transition are equal and any difference of $\sigma_{n}$ from $\sigma_{p}$ comes therefore from the Born terms only. However, both our fitting procedure on the $\mathrm{H}\left(e, e^{\prime}\right)$ data and the result of the complete calculation of refs. $[29,30]$ indicate that in the $\Delta(1232)$ region and in our kinematical conditions this non-resonant contribution accounts for less than $50 \%$ of the total strength. The overall damping observed in our and ref. [4] data at $Q^{2} \approx 0.5(\mathrm{GeV} / \mathrm{c})^{2}$ is $25 \%$ and the difference of $\sigma_{n}$ from $\sigma_{p}$ which could account for this suppression is therefore $\sigma_{n} / \sigma_{p}=0.5$. This is a too small value, which would imply that the Born terms for the neutron are completely negligible in contradiction to the basic mechanisms for the virtual photoabsorption off the nucleon, where the pion in flight contribution is sizable both for proton and neutron.

Finally, as an alternative hypothesis, we increased the non resonant background contribution in order to simulate a possible multiparticle emission which could be present in our kinematical conditions. In fact, it is well known that, as one proceeds to investigate the nuclear response in the inelastic region, the one particle-hole frame is no longer adequate, the inclusion of two particle-two hole excitations becoming more and more important [39]. We analyzed, therefore, the ${ }^{16} \mathrm{O}$ and ${ }^{12} \mathrm{C}$ measurements at $1500 \mathrm{MeV}$ where the continuous background to the resonant contribution ratio is maximized. For an enhancement of the background up to $15 \%$ we could still find a good fit to the data (comparable $\chi^{2}$ as fig. 10 results) provided that a further simultaneous damping was applied to the resonances, still supporting our previous conclusions.

\section{Conclusions}

We have measured the $\left(e, e^{\prime}\right)$ inclusive cross section on a pure ${ }^{16} \mathrm{O}$ jet target with a shower calorimeter on a wide range of energy and momentum transfer. The results in the QE region have been compared to a quite extensive calculation. Along with a realistic spectral function which contains a correlated part, the inclusion of FSI is necessary for a correct description of the data particularly at low momentum transfer. In the inelastic region, besides the Fermi motion effect, a widening and a damping of the resonances is observed in our upper limit

of $Q^{2}\left(0.2 \leq Q^{2} \leq 0.5(\mathrm{GeV} / \mathrm{c})^{2}\right)$ with respect to the single nucleon strength. In order to understand this effect, realistic calculations including medium dependent modifications as well as the difference between proton and neutron absorption should be developed. 


\section{References}

[1] P. Barreau et al., Nucl. Phys. A 402 (1983) 515.

[2] J.S. O'Connell et al., Phys. Rev. Lett. 53 (1984) 1627.

[3] D.T. Baran et al., Phys. Rev. Lett. 61 (1988) 400.

[4] R.M. Sealock et al., Phys. Rev. Lett. 62 (1989) 1350.

[5] J.S. O'Connell and R.M. Sealock, Phys. Rev. C 42 (1990) 2290.

[6] T. de Forest, Nucl. Phys. A 132 (1969) 305; E.J. Moniz, Phys. Rev. 184 (1969) 1154;

T.W. Donnelly, Nucl. Phys. A 150 (1970) 393.

[7] C. Ciofi degli Atti, E. Pace and G. Salmè, Phys. Rev. C 43 (1991) 1155.

[8] J.W. Van Orden and T.W. Donnelly, Ann. Phys. 131 (1981) 451.

[9] J.M. Laget in New Vistas in Electro-Nuclear Physics, edited by E.L. Tomusiak, H.S. Coplan and E.T. Dressler, NATO Advanced Study Institute, Series B, Vol. 142 (Plenum, New York, 1986), pp. 361-429.

[10] M. Anghinolfi et al., Proc. of the International Nuclear Physics Conference, Wiesbaden, July 1992; M. Anghinolfi et al., Phys. Rev. C 47 (1993) R922; Th. Frommhold et al., Phys. Lett 295B (1992) 28; N. Bianchi et al., Phys. Lett 299B (1993) 219.

[11] M. Taiuti et al., Nucl. Inst. and Meth. A297 (1990) 354.

[12] M. Anghinolfi et al., Nucl. Inst. and Meth. A324 (1993) 191.

[13] M. Anghinolfi et al., INFN/TC-92/15.

[14] A. Zucchiatti et al., Nucl. Inst. and Meth. A317 (1992) 492.

[15] M. Anghinolfi et al., Nucl.Inst. and Meth. A362 (1995) 292.

[16] A. Teglia, Thesis (unpublished).

[17] L. Mo and Y. Tsai, Rev. Mod. Phys. 41 (1969) 205.

[18] I. Sick et al., Nucl. Phys. A 150 (1970) 631.

[19] J.S. O'Connell et al., Phys. Rev. C 35 (1987) 1063.

[20] L. Lightbody et al., Comp. Phys., May 1988.

[21] Y. Tsai, Rev. Mod. Phys. 46 (1974) 815.

[22] R.B. Blumental et al., Phys. Rev. 144 (1966) 1199; J.G. Asbury et al., Phys. Rev. 161 (1967) 1344. 
[23] K.C. Stanfield et al., Phys. Rev. C3 (1971) 1448.

[24] C. Ciofi degli Atti, S. Simula, Phys. Lett. 325B (1994) 276.

[25] C. Ciofi degli Atti, S. Simula, L.L. Frankfurt and M.I. Strikman, Phys. Rev. C 44 (1991) R7.

[26] C. Ciofi degli Atti and S. Simula, Phys. Rev. C53 (1996) 1.

[27] T. de Forest, Nucl. Phys. A 392 (1983) 232.

[28] D.B. Day et al., Phys. Rev. C 48 (1993) 1849 and references quoted therein.

[29] S. Nozawa and T.-S.H. Lee, Nucl. Phys. A 513 (1990) 511.

[30] C.R. Chen, T. Lee, Phys. Rev. C 38 (1988) 2187.

[31] J.H. Koch and N. Ohtsuka, Nucl. Phys. A 435 (1985) 765.

[32] J. Brasse et al., Nucl. Phys. B 110 (1976) 413.

[33] W. Atwood et al., Phys. Rev. D 7 (1973) 773.

[34] R.P. Peccei, Phys. Rev. 176 (1968) 1812.

[35] R.P. Peccei, Phys. Rev. 181 (1969) 1902.

[36] G.E. Brown and W. Weise, Phys. Rev C 22 (1975) 281.

[37] M. Giannini, V. Mokeev and E. Santopinto, to appear in Int. J. Mod. Phys. E.

[38] A. Bodek and J.L. Ritchie, Phys. Rev. D 23 (1981) 1070.

[39] W. M. Alberico, M. Ericson and A. Molinari, Ann. Phys. 154 (1984) 356. 


\section{Table legend}

Tab. 1. Results of our analysis in inelastic region: $\sigma$ is the extra widening of resonances, $a$ and $b$ are the suppression factors of $\Delta(1232)$ and $\mathrm{N}(1520)$ resonances respectively, $c$ is the overall suppression factor applied to the whole inelastic contribution. 


\section{Figure legends}

Fig. 1. Ratio $R$ of the detected electrons to the luminosity for each run. The collected data correspond to $E_{e}=880 \mathrm{MeV}$ electron beam energy and $\theta_{e^{\prime}}=32^{0}$ scattering angle.

Fig. 2. Corrections applied to the spectrum at $880 \mathrm{MeV}$ beam energy and $32^{0}$ scattering angle. The experimental data (open triangles) and the radiatively corrected data (black square) are shown together with the elastic radiative tail (full curve), the hard photon emission before (dot-dashed) and after (dotted) nuclear scattering and the pair production contribution (dashed). The soft photon emission correction is not reported.

Fig. 3. Comparison of ${ }^{16} \mathrm{O}\left(e, e^{\prime}\right)$ inclusive cross section measured in the present experiment (open squares) with the results of ref. [19] (full dots) at $540 \mathrm{MeV}$ electron beam energy and $37.1^{0}$ scattering angle.

Fig. 4. Inclusive cross section measured for the process ${ }^{16} \mathrm{O}\left(e, e^{\prime}\right)$ versus the energy transfer $\omega$ in three kinematical conditions corresponding to the electron scattering angle $\theta_{e^{\prime}}=32^{0}$ and incident energy a) $E_{e}=700$, b) 1080 and c) $1200 \mathrm{MeV}$. The dashed lines represent the theoretical predictions obtained within the IA, the solid lines include the effects of the FSI of the knocked-out nucleon with the residual nuclear system evaluated according to ref. [26]. On the top of each plot the value of the Bjorken scaling variable $x$ is also reported.

Fig. 5. Experimental inclusive cross section in $a, b){ }^{16} \mathrm{O}$ (this experiment) and $c, d$ ) ${ }^{12} \mathrm{C}[4]$ in different kinematical conditions. The data are compared with the calculation (full curve), which includes the QE contribution (dotted) as well as the inelastic strength directly deduced from the free proton (long dashed).

Fig. 6. The inclusive cross section per nucleon normalized to the virtual photon flux vs. the invariant mass $W$. The open squares and full circles are the data for ${ }^{16} \mathrm{O}$ and ${ }^{12} \mathrm{C}$; the $Q^{2}$ value at the $\Delta(1232)$ peak is 0.25 and $0.26(\mathrm{GeV} / \mathrm{c})^{2}$, respectively.

Fig. 7. Example of the $\mathrm{H}\left(e, e^{\prime}\right)$ cross section data [4] fitted with our version of the Brasse [32] parametrization (full curve); the dashed lines represent the resonant and non-resonant contributions to the cross section as determined in our analysis.

Fig. 8. Inclusive $\left(e, e^{\prime}\right)$ cross section in $\left.a, b\right){ }^{16} \mathrm{O}$ (this experiment) and $\left.c, d\right){ }^{12} \mathrm{C}$ (ref. [4]). The different contributions to the inelastic cross section are shown: the non-resonant background (dotted), the $\mathrm{D}(1232)$ and the $\mathrm{N}(1520)$ resonances (dashed curves). At this step of the analysis the resonance cross-section was broadened by the additional width reported in table I. The full curve represents the sum of this parametrization and the QE contribution.

Fig. 9. Final result of our analysis (QE + inelastic), where an extra widening of the resonant part and a suppression factor is applied to the $\Delta(1232)$ and $\mathrm{N}(1520)$ resonances only (thin curve) or to all the inelastic contribution (thick curve). The corresponding suppression factors are reported in table I.

Fig. 10. The suppression factors as deduced from our analysis for ${ }^{16} \mathrm{O}$ (full squares)

and ${ }^{12} \mathrm{C}$ (open circles) as a function of $Q^{2}$ at fixed invariant mass $W$. The result of the analysis [37] at the photon point is also reported (open triangles). 


\section{Table 1}

\begin{tabular}{|c|c|c|c|c|c|}
\hline & $\begin{array}{c}E_{0}, \theta \\
(\mathrm{MeV}, \mathrm{deg})\end{array}$ & $\begin{array}{c}\sigma \\
(\mathrm{MeV})\end{array}$ & $a$ & $b$ & $c$ \\
\hline \hline${ }^{16} \mathrm{O}$ & 700,32 & $50 \pm 15$ & $1.00 \pm 0.05$ & - & $1.00 \pm 0.05$ \\
\cline { 2 - 6 } & 880,32 & $50 \pm 15$ & $0.90 \pm 0.05$ & - & $0.90 \pm 0.05$ \\
\cline { 2 - 6 } & 1080,32 & $50 \pm 15$ & $0.95 \pm 0.07$ & - & $0.95 \pm 0.07$ \\
\cline { 2 - 6 } & 1200,32 & $75 \pm 15$ & $1.00 \pm 0.07$ & $0.8 \pm 0.1$ & $0.95 \pm 0.07$ \\
\cline { 2 - 6 } & 1500,32 & $80 \pm 25$ & $0.50 \pm 0.08$ & $0.4 \pm 0.2$ & $0.75 \pm 0.07$ \\
\hline${ }^{12} \mathrm{C}$ & $960,37.5$ & $50 \pm 10$ & $0.85 \pm 0.04$ & - & $0.90 \pm 0.04$ \\
\cline { 2 - 6 } & $1100,37.5$ & $75 \pm 10$ & $0.85 \pm 0.04$ & - & $0.90 \pm 0.04$ \\
\cline { 2 - 6 } & $1200,37.5$ & $60 \pm 10$ & $0.80 \pm 0.04$ & $0.8 \pm 0.1$ & $0.90 \pm 0.04$ \\
\cline { 2 - 6 } & $1500,37.5$ & $50 \pm 10$ & $0.60 \pm 0.04$ & $0.4 \pm 0.2$ & $0.80 \pm 0.04$ \\
\hline
\end{tabular}




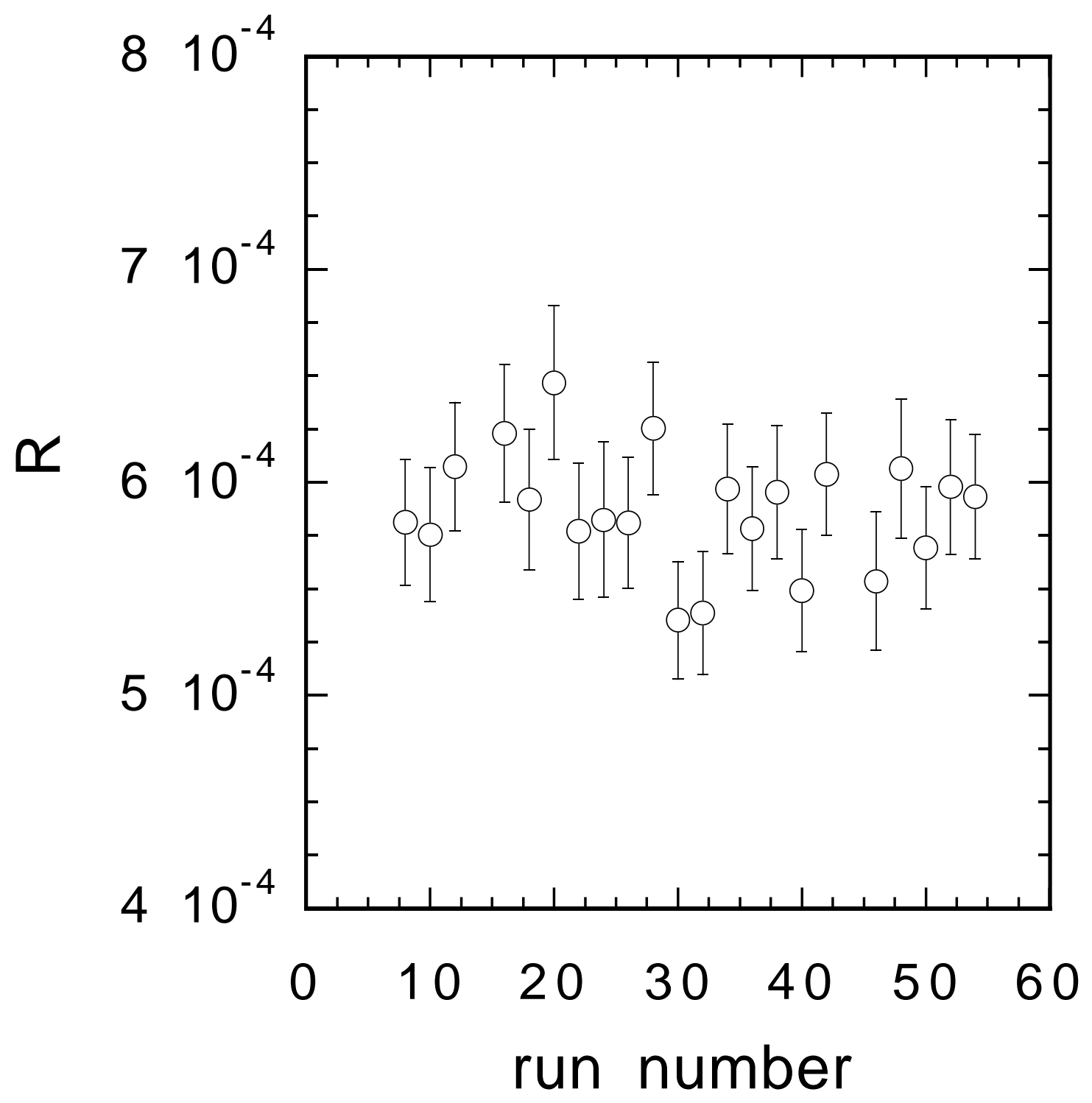

M. Anghinolfi et al., Nucl. Phys. A: fig. 1. 


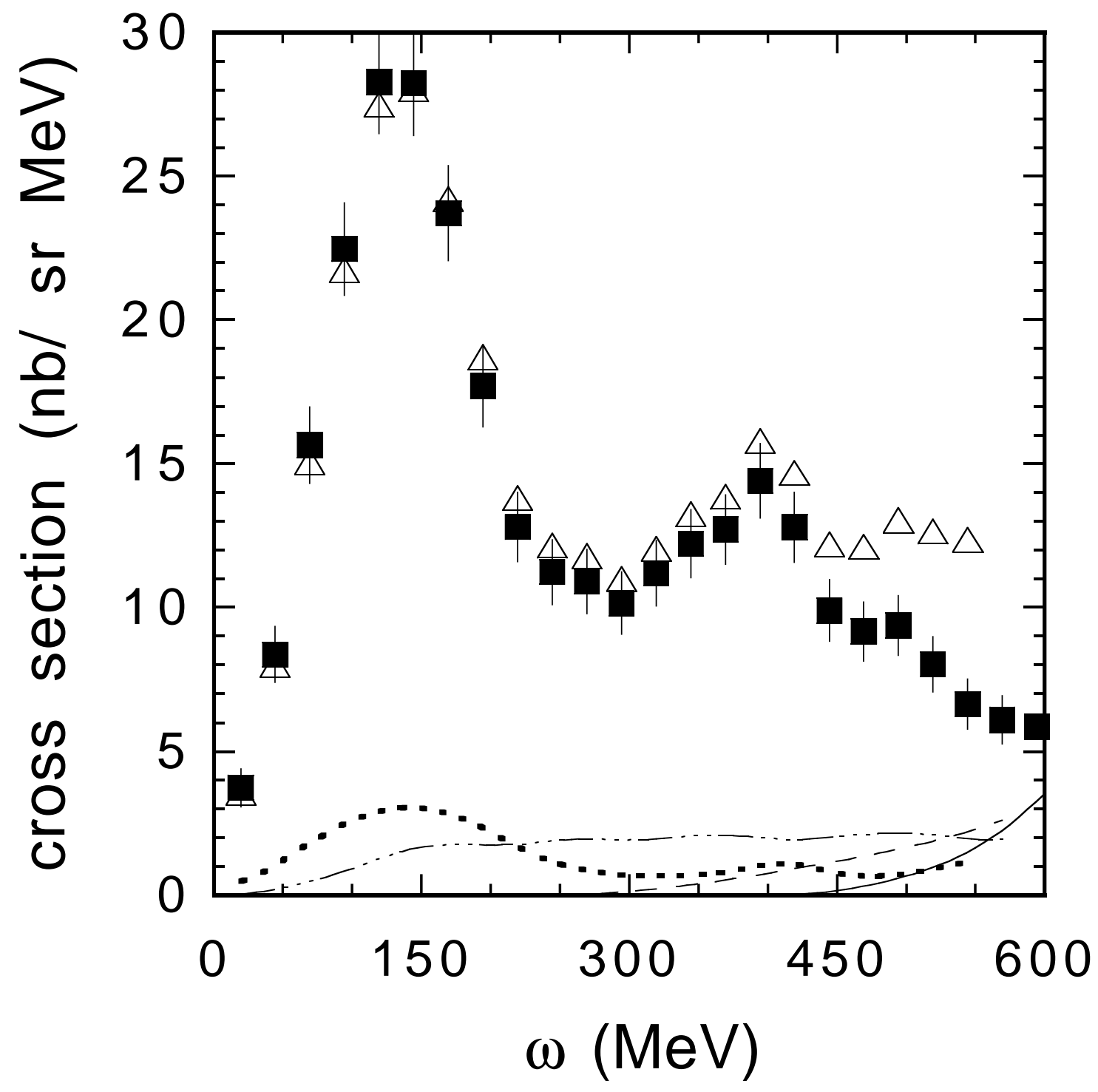

M. Anghinolfi et al., Nucl. Phys. A: fig. 2. 


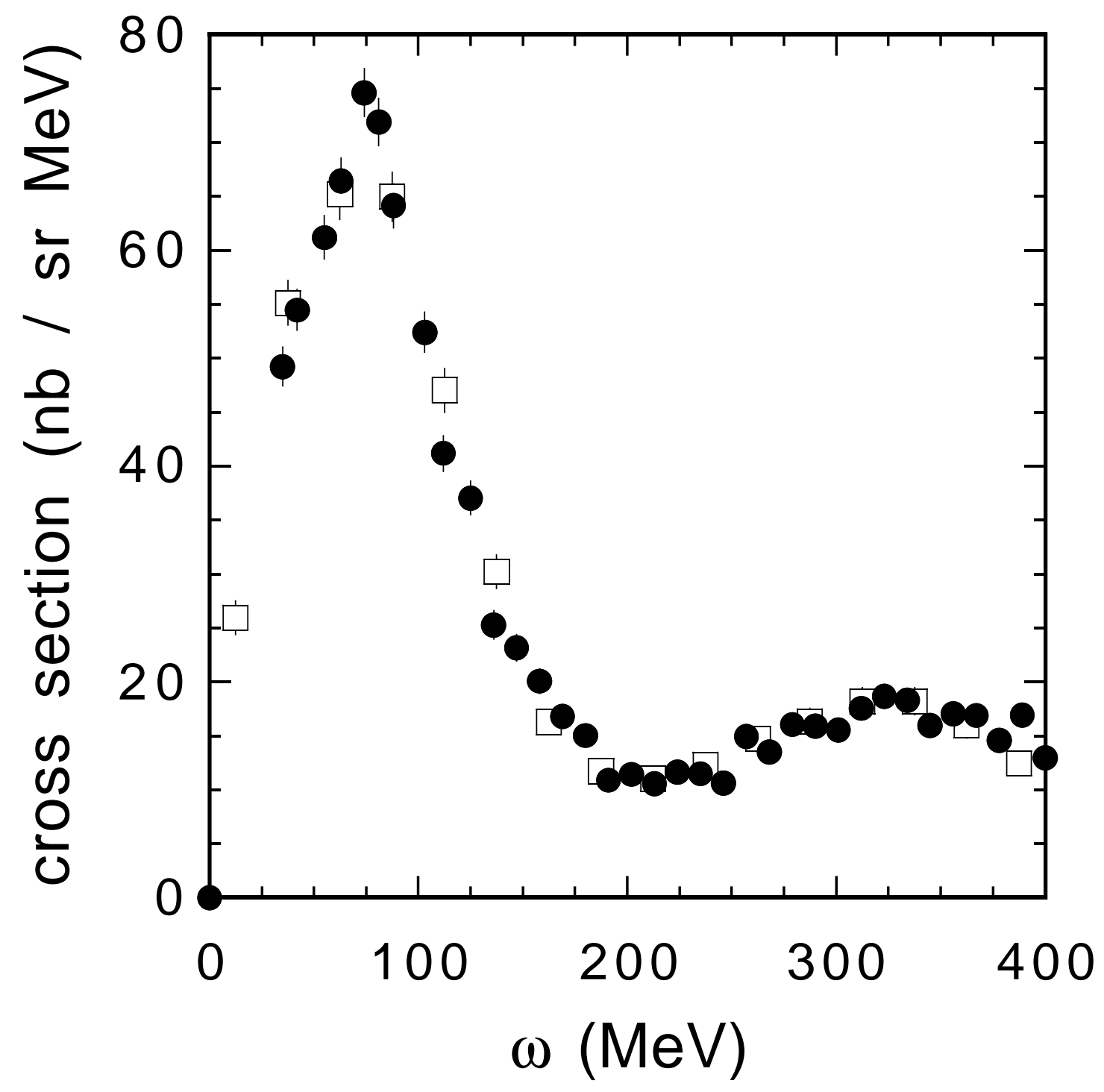

M. Anghinolfi et al., Nucl. Phys. A: fig. 3. 

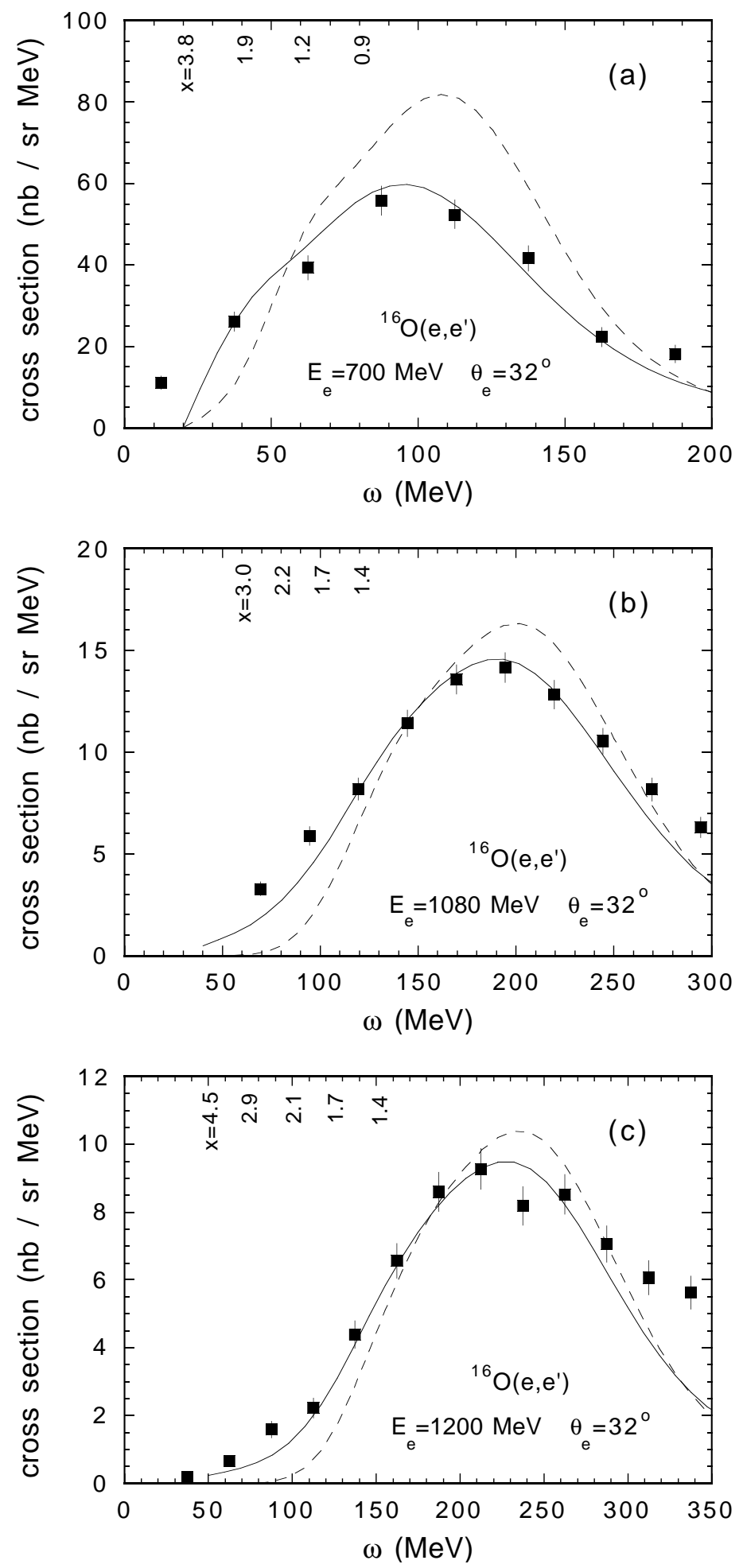

M. Anghinolfi et al., Nucl. Phys. A: fig. 4. 

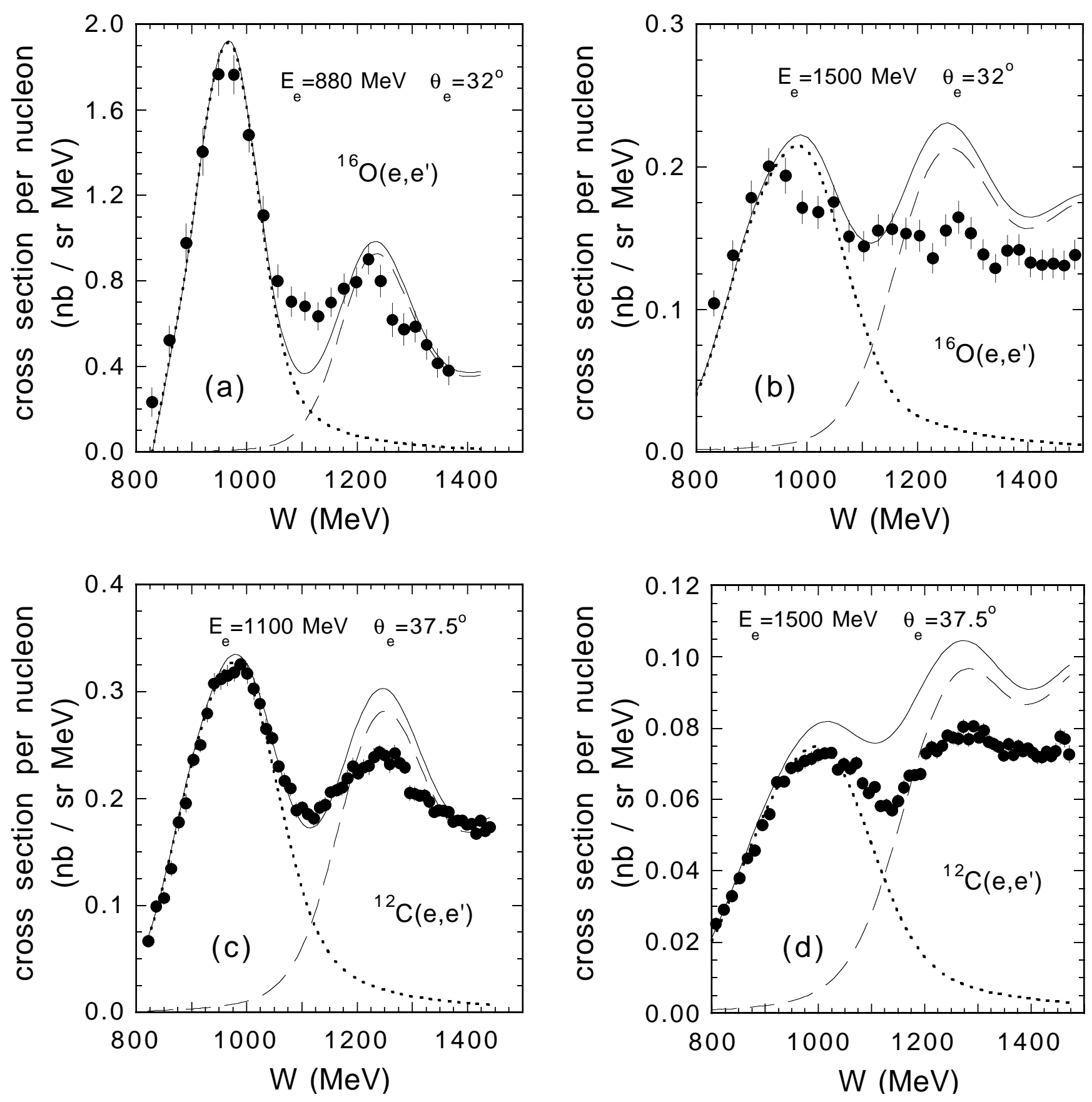

M. Anghinolfi et al., Nucl. Phys. A: fig. 5. 


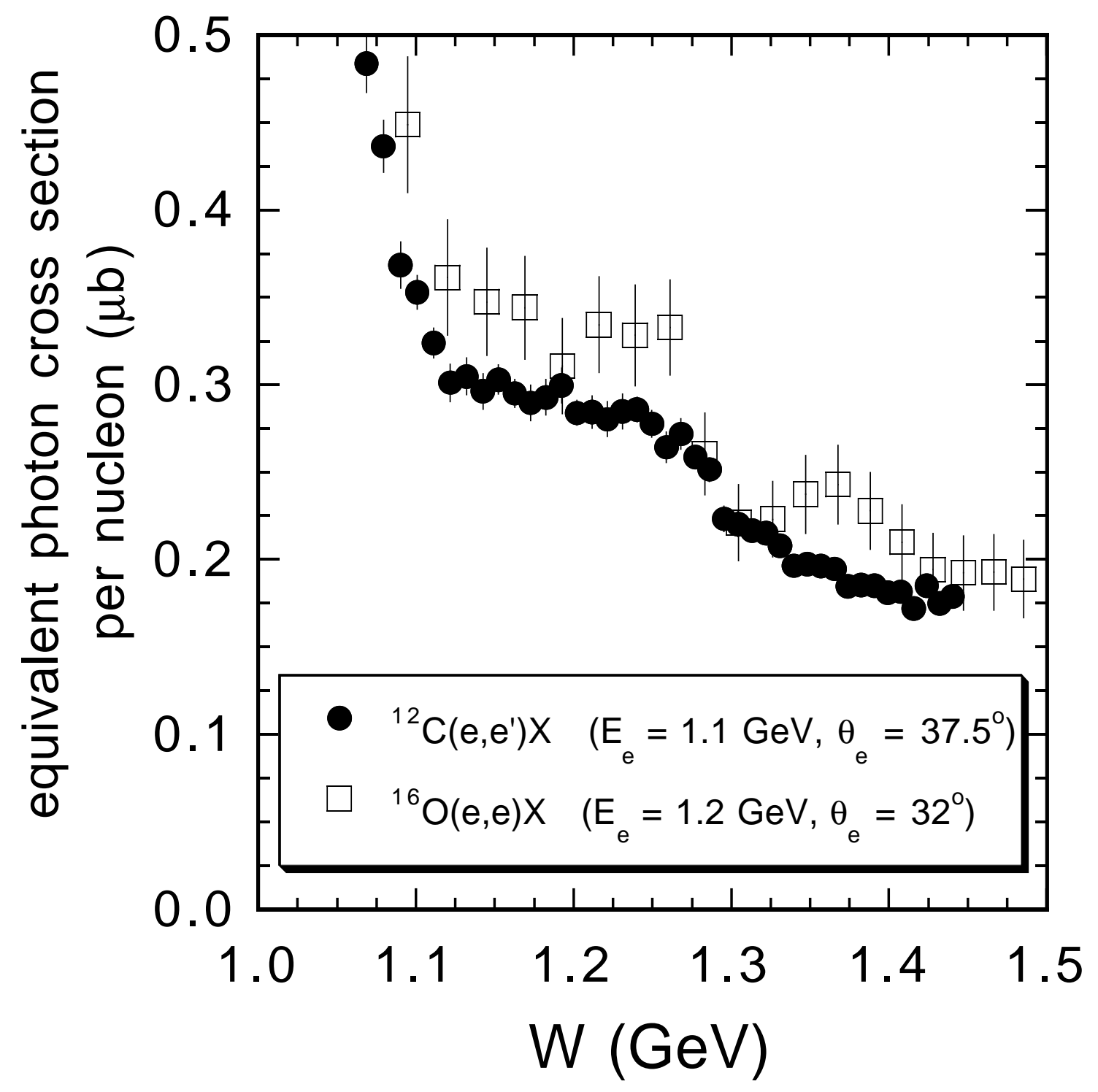

M. Anghinolfi et al., Nucl. Phys. A: fig. 6. 


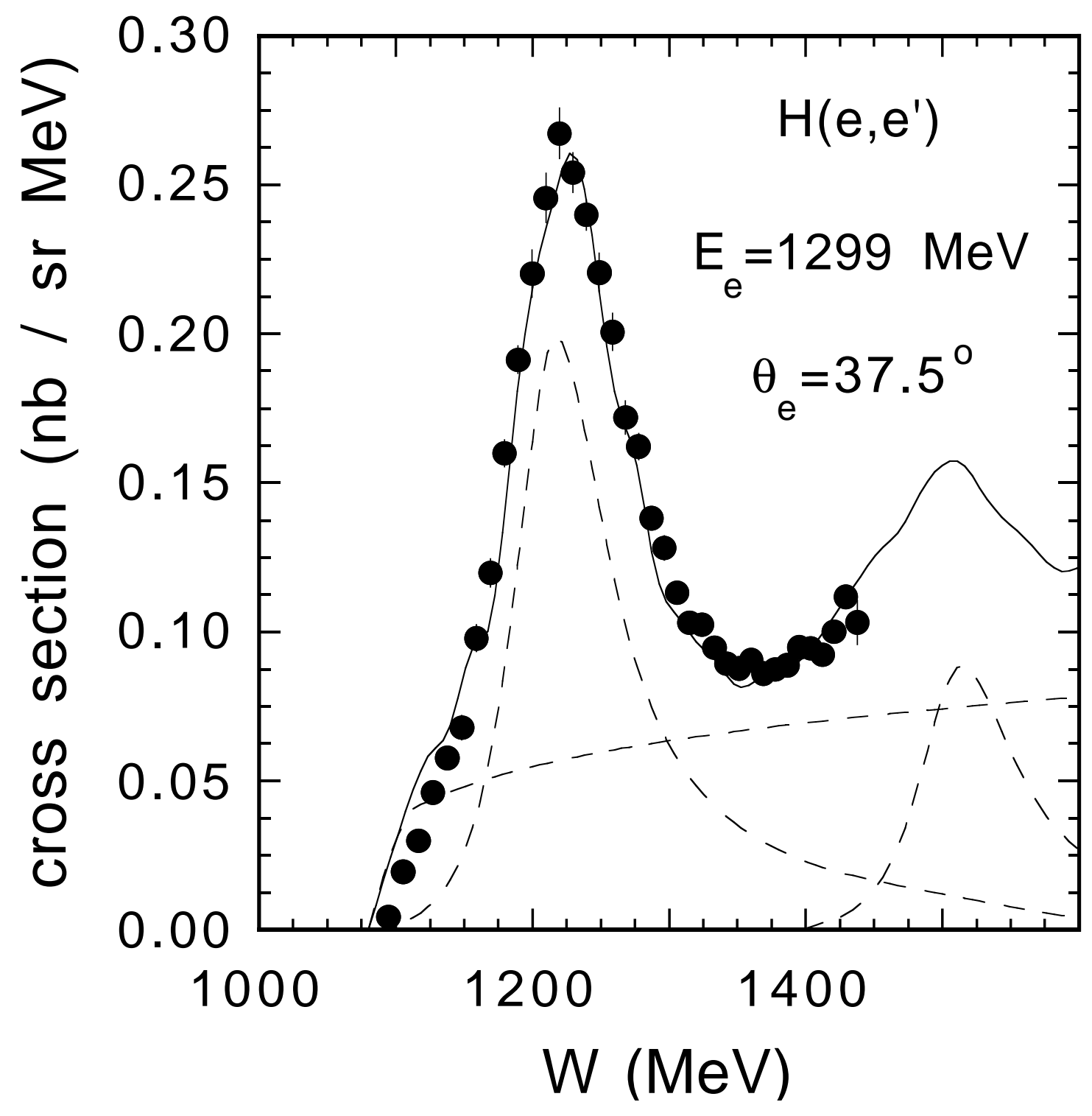

M. Anghinolfi et al., Nucl. Phys. A: fig. 7. 

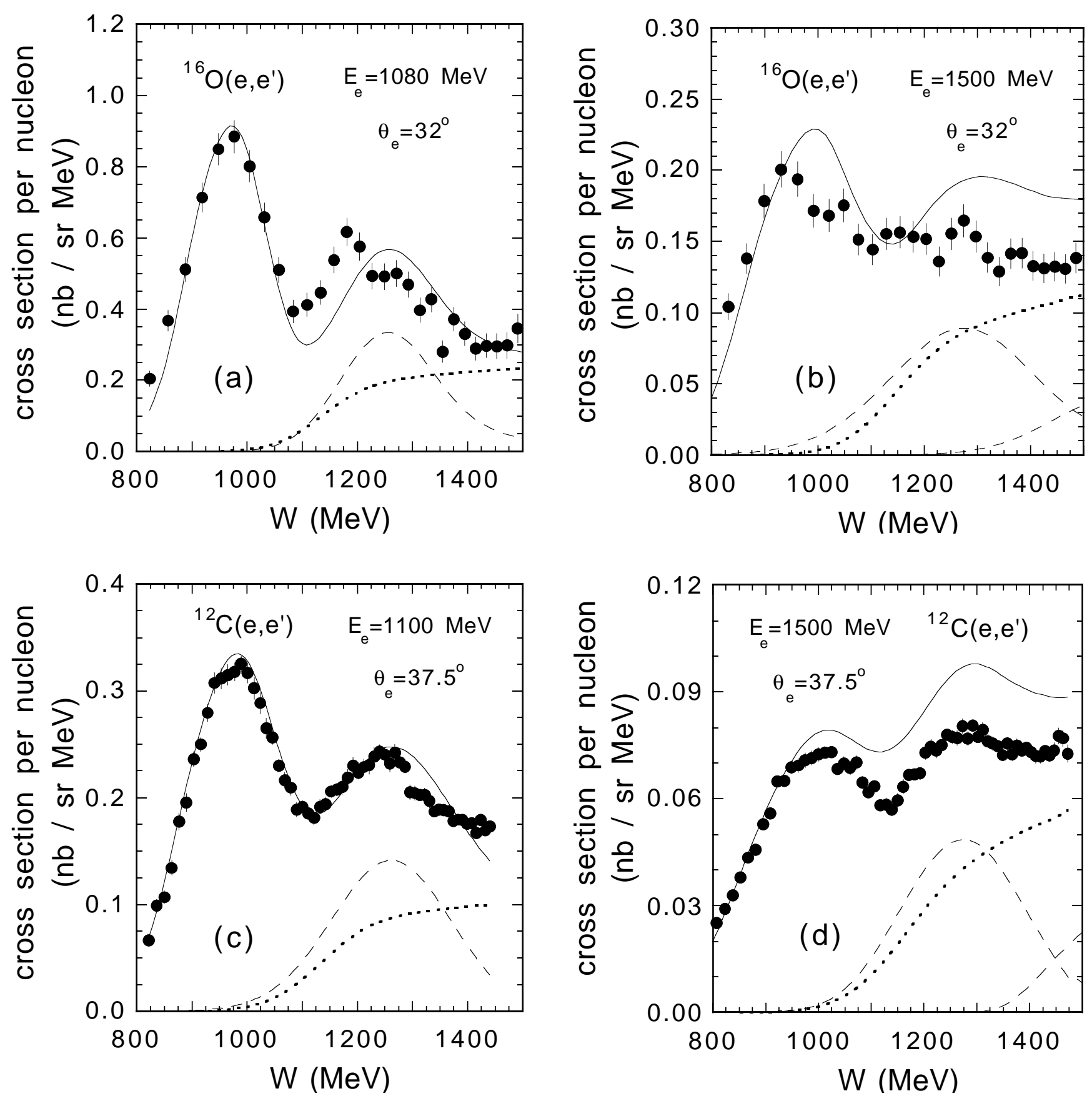

M. Anghinolfi et al., Nucl. Phys. A: fig. 8. 

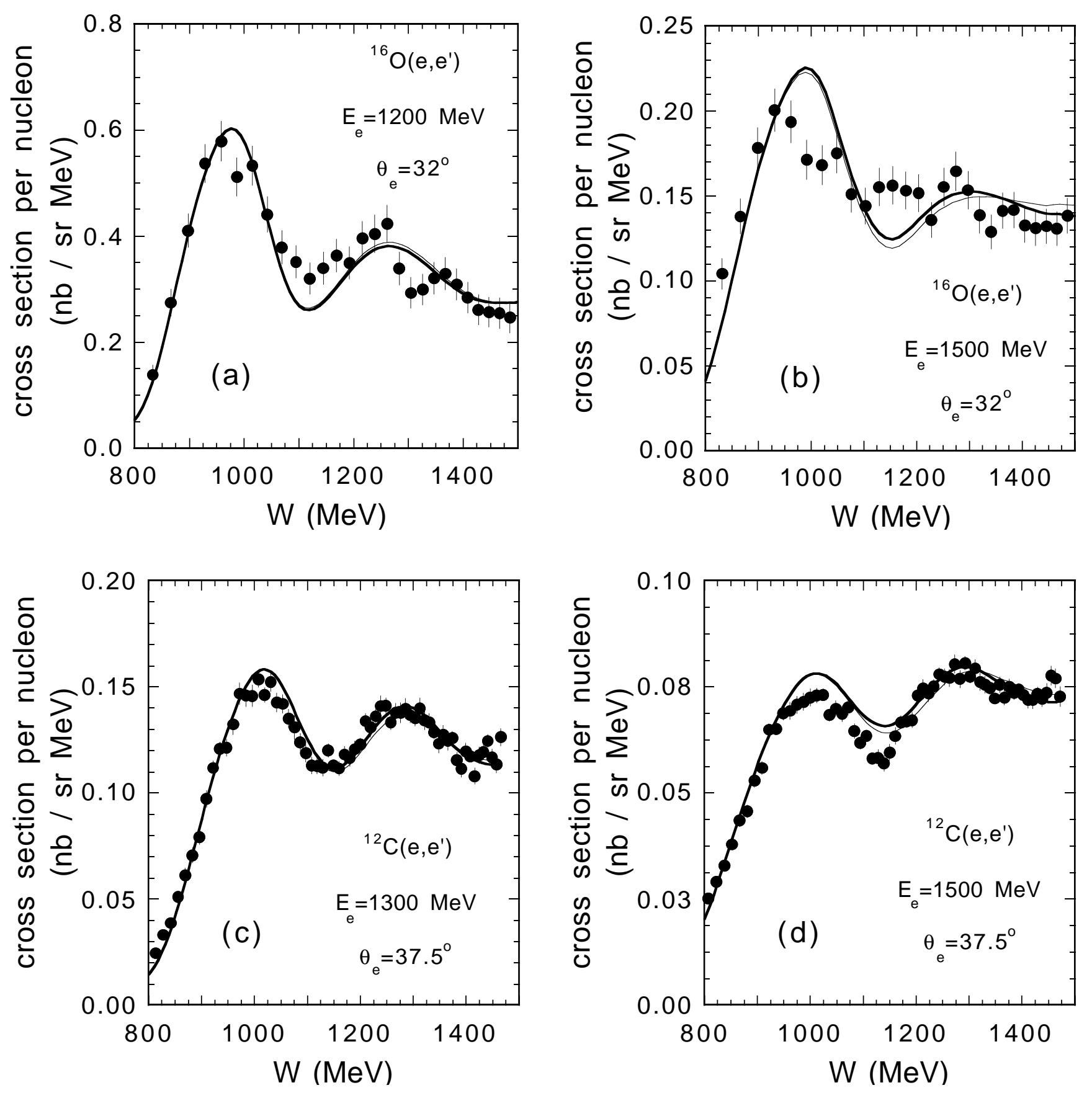

M. Anghinolfi et al., Nucl. Phys. A: fig. 9. 

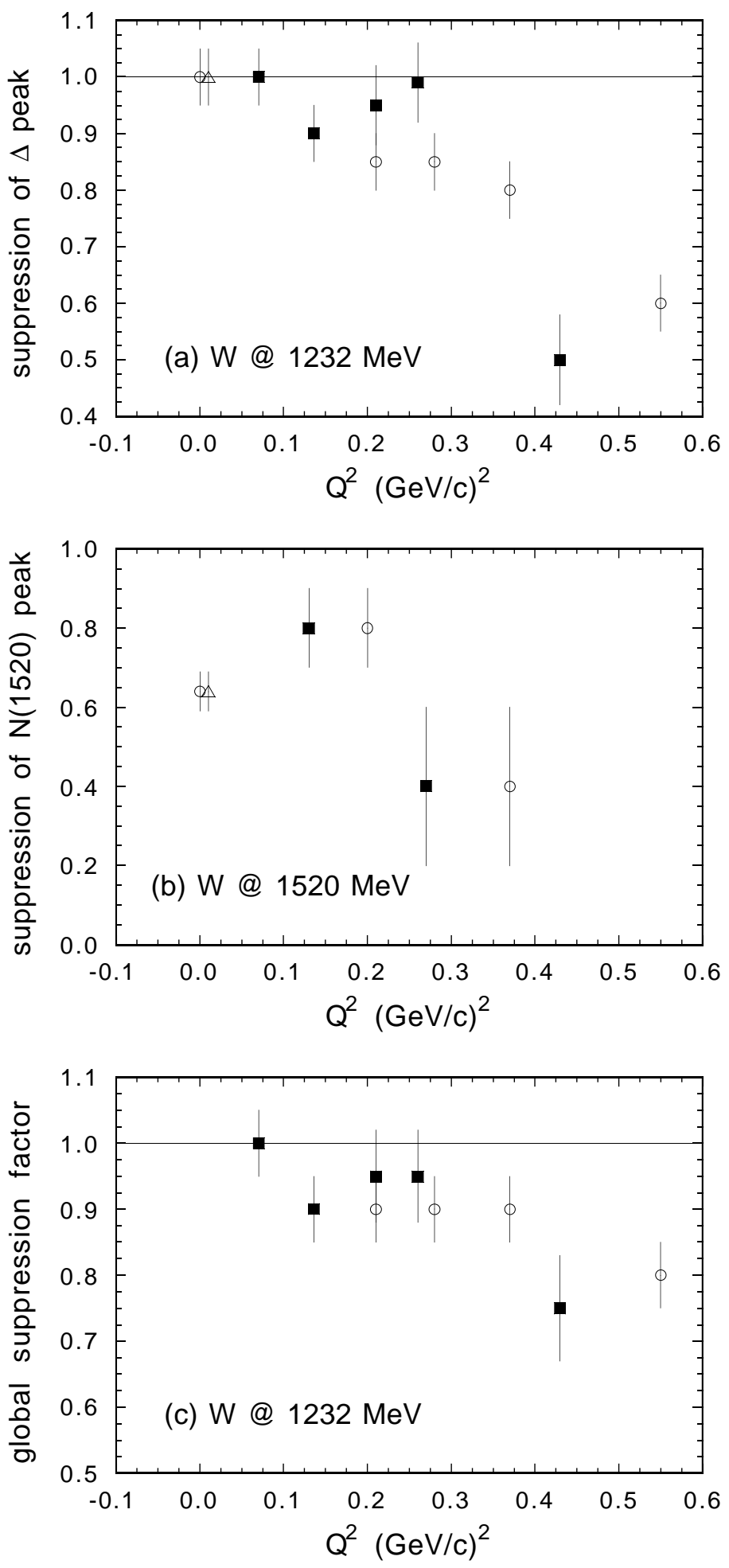

M. Anghinolfi et al., Nucl. Phys. A: fig. 10. 